\title{
A Novel Radon Transform and AR Model in Crack Detection
}

\author{
Siming Yang ${ }^{1}$, Peng Xue ${ }^{2, *}$, Ao Hong ${ }^{3}$, Zhou Bo ${ }^{3}$, Haicheng Liu ${ }^{1}$ and Mingri \\ Cong $^{1}$ \\ ${ }^{1}$ Information Science and Technology Institute, Zhengzhou, China \\ ${ }^{2}$ Naval Aeronautical and Astronautical University, Yantai, China \\ ${ }^{3}$ Xichang Satellite Launch Center, Xichang, China \\ *Corresponding Author: Peng Xue (Email:xuep04@foxmail.com)
}

\section{Keywords: Radon Transform; AR model; Crack Detection}

\begin{abstract}
Aiming at the peak extraction problem in the radon space, a peak extraction method based on AR model is proposed to detect component crack value. A profile analysis technique is used to take profile in Radon space and reconstruct profile with AR model to get a sharp and prominent peak for crack value extraction. Intensive experiments have shown that the proposed method is effective in estimating the component crack and lessening peak extraction difficulty.
\end{abstract}

\section{Introduction}

The crack parameter is an important parameter to ensure equipment operates safely and stably, and crack measurement is a main part in assembly and checkout, different levels feeler is commonly used to detect different crack value, and it's inconvenient to determine the crack.

Detection technology based on image processing setting optical, computer technology and the digital image processing technology as a whole, can be used for the dimensional measurements of geometric quantities. It has the advantages of non-contact, high speed, high precision, widely used in various measurement occasions [1]. The main problem in crack measurement is the line detection and recognition, line detection can be instead by point detection with the Radon transform, lessen the line recognition difficulty greatly. But affected by Image noise, and interference lines, it's also very difficult to extract the target point in Radon space.

Zhang Qiao-ping and Isabelle Couloigner considered that the point whose value is half of the maximum point corresponds to the edge line of a crack, and obtain the crack value [2]. An algorithm is proposed to extract blood vessels by classify vessels into some predefined class according to vessel width and compute for each class. The width examination algorithm is pointed out in [2, 3], but the peaks detection method have not point out clearly. HAO Ying propose an improved algorithm for peak detection based on the basic principles of the Forstner operator in [4]. An algorithm developed for ship wakes detection makes decision in feature space from [5]. And in [6], the difference between the real peaks and interference peaks is magnified by a local operator. The emphasis in $[4,5,6]$ is image reconstruction and restoration, and has no crack calculation method. This paper proposes a new method based on AR model to detect the peaks in Radon space and obtain the crack value.

\section{RADON TRANSFORMATION PRINCIPLES}

Radon transform is a mathematical basis for image reconstruction defined as the (1) by Johann Radon in 1917 for the first time, whose principle is that the Radon transform maps pixels set in the image space to a single point in the Radon space, $\theta$ represents the angle between the line and the $\mathrm{x}$-axis, ${ }^{\rho}$ is the length from the origin to the straight line. Radon transform for each angle woven together to appear as an angle and Radon value figure, angle range from $0^{\circ} \sim 179^{\circ}$ in $1^{\circ}$ increments, the strength values corresponding to each angle in the original image is the integral along each direction straight line. Each point in $(\rho, \theta)$ Space corresponds to the a straight line in original image, 
therefore, a high gray value line form a bright point , and the line detection in the image space will be simplified as the peak detection in the transform space[7].

The radon transform in 2-D Euclidean space is defined by

$$
R(\rho, \theta)=\iint_{D} f(x, y) \delta(\rho-x \cos \theta-y \sin \theta) d x d y
$$

Where $\mathrm{D}$ is the whole image, $f(x, y)$ is the gray level of image at position $(x, y), \delta$ denotes the Dirac delta function, $\theta$ represents the angle in polar coordinates, $\rho$ is the length from the origin to the straight line. The Dirac delta function forces the integration of $f(x, y)$ along the line [8-9].

\section{AR MODEL}

Parameter model is the main content of modern spectral estimation, include full pole model (AR model), full zero models (MA model) and pole-zero model (ARMA model).

AR model had the properties of auto regression, the output now is the weighted sum of the input now and the past p output. Models can be expressed as follow difference equation:

$$
\begin{aligned}
& x(n)=-\sum_{k=1}^{p} a_{k} x(n-k)+u(n) \\
& H(z)=1 /\left(1+\sum_{k=1}^{p} a_{k} z^{-k}\right)
\end{aligned}
$$

Where $\mathrm{p}$ is the order of the model and the output $x(n)$ is produced by exciting a p-order AR Linear System $H(z)$ from a white noise $u(n)$ with variance $\sigma^{2}$. Since $H(z)$ is a linear system, the power spectrum of $x(n)$ as follows:

$$
P_{x}\left(e^{j w}\right)=P_{u}\left(e^{j w}\right)\left|H\left(e^{j w}\right)\right|^{2}=\sigma^{2} /\left|1+\sum_{k=1}^{p} a_{k} e^{-j w k}\right|^{2}
$$

If the white noise variance $\left(\sigma^{2}\right)$ and the model parameters $\left(a_{k}\right)$ is given, and $u(n), x(n)$ are stationary random signal, Power spectrum of the output sequence ${ }^{x(n)}$ can be obtained from the above equation. The canonical equation (5) can be established by the relationship between the AR model parameters and the autocorrelation function.

$$
\left[\begin{array}{ccccc}
r_{x}(0) & r_{x}(1) & r_{x}(2) & \cdots & r_{x}(p) \\
r_{x}(1) & r_{x}(0) & r_{x}(1) & \cdots & r_{x}(p-1) \\
r_{x}(2) & r_{x}(1) & r_{x}(0) & \cdots & r_{x}(p-2) \\
\vdots & \vdots & \vdots & \ddots & \vdots \\
r_{x}(p) & r_{x}(p-1) & r_{x}(p-2) & \cdots & r_{x}(0)
\end{array}\right]\left[\begin{array}{c}
1 \\
a_{1} \\
a_{2} \\
\vdots \\
a_{p}
\end{array}\right]=\left[\begin{array}{c}
\sigma^{2} \\
0 \\
0 \\
0 \\
0
\end{array}\right]
$$

Where $r_{x}(m)$ is the autocorrelation function of $x(n), a_{k}$ is the model parameters. From (5), the model parameters and variance $a_{1}, a_{2}, a_{3} \cdots a_{p}, \sigma^{2}$ can be obtained from the autocorrelation function $r_{x}(0), r_{x}(1), r_{x}(2) \cdots r_{x}(p)$ in a p-order AR model, and the power spectrum can be estimated by (4) [10-11].

\section{PROFILE SIGNAL RECONSTRUCTION WITH AR MODEL}

The power spectrum is the concept of statistical average of the random process, the power spectrum of the stationary random process is a definite function, therefore, the profile signal can be taken as power spectrum of stationary random signal, and reconstruct signal with AR model, Steps as follows:

Step1, Do Radon transformation, and extract profile signal.

Step2, Do Inverse Fourier transform to obtain correlation function of AR model.

Step3, Select AR model order (in this paper $\mathrm{p}=25$ ), and construct the canonical equation.

Step4, Obtain the AR model parameter $a_{1}, a_{2}, a_{3} \cdots a_{p}, \sigma^{2}$.

Step5, Reconstruct the profile signal according to (4). 
Step6, Calculating crack from the peak position in reconstructed image, namely the two peak position difference value.

\section{PERFORMANCE TEST AND RESULT ANALYSIS}

This paper applied algorithm to 17 images taken from a component, the image dimensions are $1280 \times 1024$, the testing environment is that HP desktop whose dominant frequency is $2.62 \mathrm{GHz}$ and memory is 2G, Windows XP system and Matlab7.8.The result is shown in Fig. 3.

Fig. 3(c) shows the signal fitted by Gaussian, Fig. 3(d) shows the signal reconstructed by AR model. As can be seen from Fig.3, signal fitted by Gaussian has an obvious peaks, but the peak location has deviation, and also has no sharp and prominent peaks; Signal reconstructed by AR model has sharp, smoothing and prominent peaks, and also easy to locate peaks.

The cracks obtained from each new signal are shown in Tab. I. It's clear that the proposed algorithm is capable of estimating an accurate crack value based on the radon transform, the mean of the errors is 0.24 pixels and the variance is only 1 . It's accurate enough for most application.
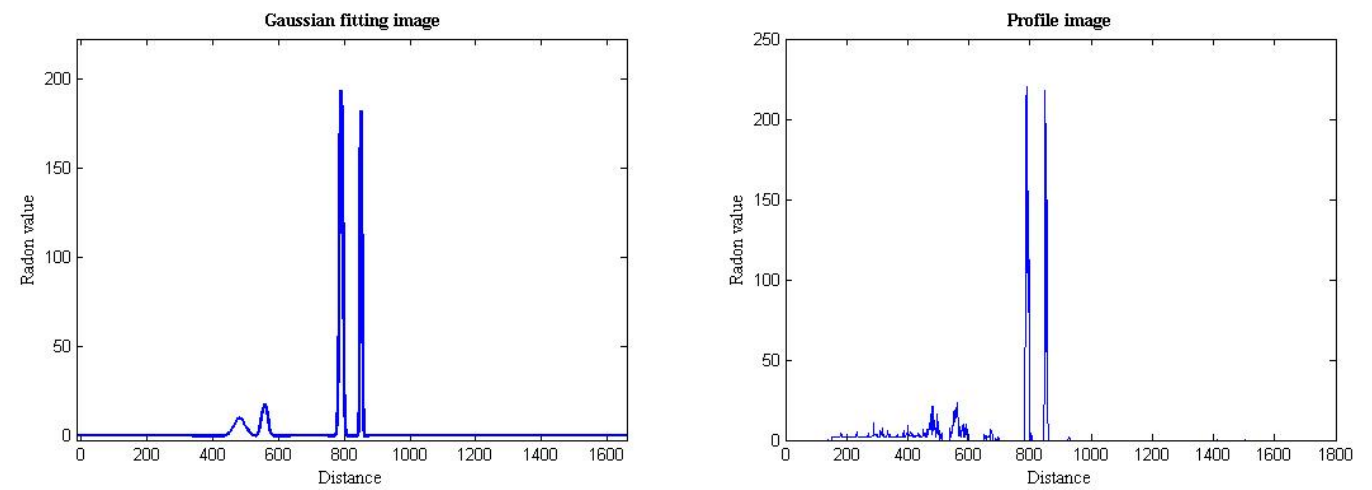

Figure 1. The result of profile reconstruction:and AR model reconstruct image

\section{Conclusion}

This paper apply AR model to reconstruction profile signal taken along the angle which include maximum value in Radon space, and locating peaks to obtain crack value. Result shows that the reconstructed signal by AR model has sharp, smoothing and prominent peaks, and also easy to locate peaks in Radon space, and then extract straight line to obtain crack value. Because the reconstruct quality effected by the order of AR model, if low, the reconstructed signal will be too smooth, on the opposite, false peaks may appear, this resulted in the best model parameter is difficult to determine, But AR model will be widely used to detect peaks in Radon space with the model order selection algorithm improve and be capable to meet testing automation of component crack detection.

\section{References}

[1] Jiang jia-dong,Yuan dao-cheng.Research on on-line-measuring clearance of assembly parts based on image measurement technology J. Journal of transducer technology, China,2005, vol.24, no.12, pp. 26-28.

[2] Qiaoping Zhang,Isabelle Couloigner. “Accurate Centerline Detection and Line Width Estimation of Thick Lines Using the Radon Transform ” J. IEEE Transactions on Image Processing, 2007, vol.16,no.2,pp.310-316.

[3] Reza Pourreza,Touka Banaee,Hamidreza Pourreza. “A Radon Transform Based Approach for Extraction of Blood Vessels in Conjunctival Images” J. Lecture Notes in Computer Science,2008, vol.5317,no.1,pp.948-956. 
[4] Hao Ying, Wang Li-qiang. “An Improved Algorithm of Forstner Operator in the Application of Peaking Value Measurement in the Radon Transform” J. Microelectronics \& Computer, China, 2008, vol.25,no.12,pp.190-193.

[5] Chen Zhen-lin, Zou Huan-xin. "Detection and Discrimination Method of Ship Wakes in SAR Imagery ” J. Modern Radar, China, 2010, vol.3,no.21,pp.42-47.

[6] Ji Jun-Hong, Chen Guo-dong. "Improved Hough transform based on peak detection by local operators” J. Application Research of Computers, China, 2008, vol.2,no.55,pp.1569-1572.

[7] Yang gao-bo, Du qing-song. Matlab image video processing applications and example M.China,Electronic Industry Press, China, 2010, pp.89-92.

[8] Huixian Duan,Yuxi Zhang. "An improved method of ship wake detection based on localized Radon transform” C. IET International Radar Conference 2009.Guilin:128.

[9] Wang Li,Chang Qing.“ Radon transform for line segment detection in low SNR image” J. Infrared and Laser Engineering, China, 2003, vol.32,no.2,pp.163-166.

[10] Hu guang-shu.Digital signal processing M.China,Tsinghua university press , 2010,pp.527-546.

[11] Wei Wei, Qi Yong. Information potential fields navigation in wireless Ad-Hoc sensor networks[J]. Sensors, 2011, 11(5): 4794-4807..

[12] Wei W, Xu Q, Wang L, et al. GI/Geom/1 queue based on communication model for mesh networks[J]. International Journal of Communication Systems, 2014, 27(11): 3013-3029. 